\title{
Assessment of Severity of Dengue Fever by Deranged Alanine Aminotransferase Levels
}

\author{
Fatima Ayaz $^{1}$, Muhammad Furrukh ${ }^{1}$ \\ 1. Department of Medicine, Holy Family Hospital, Rawalpindi, PAK
}

Corresponding author: Muhammad Furrukh, furrukhhh@gmail.com

\begin{abstract}
Introduction

Dengue fever (DF) is the most common arbovirus-related infection worldwide. Most of the dengue infections are asymptomatic. The clinical presentation of DF varies from mild febrile illness to dengue hemorrhagic fever (DHF) and dengue shock syndrome (DSS). Both DHF and DSS are severe forms of illness and carry higher rates of mortality. Various clinical parameters are associated with severe DF. The objective of this study was to determine any relation between elevated alanine aminotransferase (ALT) levels at presentation and development of severe DF.
\end{abstract}

\section{Methods}

This cross-sectional study was carried out at a large tertiary care hospital in Rawalpindi, Pakistan. Over a period of six months, 65 dengue patients were enrolled and their ALT levels were recorded at presentation. All the patients were managed as per guidelines in a similar way, and clinical course was followed for the development of severe forms of DF, such as DHF and DSS.

\section{Results}

Out of total 65 patients, 45 (69.2\%) were males and 20 (30.8\%) were females. Classical DF was present in 18 (27.7\%) patients, whereas 47 (72.3\%) patients developed DHF or DSS. Patients with DF had a mean ALT level of $131.67( \pm 244.48) \mathrm{U} / \mathrm{L}$ at presentation, whereas patients with DHF and DSS had a mean ALT level of $228.15( \pm 467.88) \mathrm{U} / \mathrm{L}$ at presentation. The Mann-Whitney U test was applied to compare differences between ALT levels of both groups, and p value was found to be $0.018(<0.05)$. Thus, it was concluded that there was a statistical difference between ALT levels of patients with simple DF and patients with DHF or DSS.

\section{Conclusion}

Higher serum levels of ALT at presentation can give a clue about possibility of progression to severe forms of DF (DHF and DSS). Therefore, patients with higher ALT at presentation should be prioritized and monitored more rigorously.

Received 09/04/2020

Review began 09/06/2020

Review ended 09/14/2020

Published 09/19/2020

(c) Copyright 2020

Ayaz et al. This is an open access article distributed under the terms of the Creative Commons Attribution License CC-BY 4.0., which permits unrestricted use, distribution, and reproduction in any medium, provided the original author and source are credited.

Categories: Internal Medicine, Gastroenterology, Infectious Disease

Keywords: dhf, dss, alt, dengue fever, alanine aminotransferase, dengue hemorrhagic fever, dengue shock syndrome

\section{Introduction}

Dengue fever (DF) is the most common arbovirus borne infection worldwide, and its incidence is increasing continuously throughout the globe [1]. Approximately, 50-100 million cases occur annually with around 10,000 dengue deaths per annum [2].

Most of the dengue infections are asymptomatic. The clinical presentation of DF varies from mild febrile illness to dengue hemorrhagic fever (DHF) and dengue shock syndrome (DSS) [3]. The classical DF is mild, febrile illness, while on the other hand DHF is characterized by high-grade fever, increased vascular permeability, plasma leakage, and hemorrhagic manifestations which if accompanied by shock is termed as DSS. Both DHF and DSS are severe forms of illness and carry higher rates of mortality [4]. Various clinical parameters and laboratory investigations, such as leukocyte count, prothrombin time, activated partial thromboplastin time, alanine aminotransferase (ALT) levels, aspartate aminotransferase levels, and hematocrit levels, have been studied internationally to forecast severity of infection [3,5]. Liver is not the major target organ of dengue virus, but hepatic involvement can occur due to a direct viral effect on hepatic cells or due to dysregulated immune response of the body [6,7].

In past, many national and international studies have been done to evaluate the role of liver function tests in forecasting the severity of DF. However, there was a paucity of data, pertaining specifically to relationship between elevated ALT levels and development of DHF and DSS. Early identification of DHF and DSS patients can be very crucial in resource-limited setups as these patients can be saved by strict monitoring and early 
aggressive management. ALT levels being widely available and inexpensive can be very useful in such less resourceful setups. Therefore, this study was designed with an objective to assess the utility of ALT as a marker of severe DF.

\section{Materials And Methods}

This cross-sectional study was carried out at the Department of Infectious Diseases of Holy Family Hospital, Rawalpindi. The duration of the study was six months (from October 2019 to March 2020).

\section{Inclusion and exclusion criteria}

Adult patients with confirmed DF as per definition of Dengue Expert Advisory Group, Punjab, Pakistan (DEAG) were included in the study [8]. However, patients with known history of chronic liver disease and patients with history of known hepatotoxic drug use within last six months were excluded from the study.

\section{Operational definitions}

DF was defined as fever of 2-12 days with at least two of the following: headache, retro-orbital pain, arthralgias/backache, myalgias, abdominal pain, rash, bleeding, and decreased urine output along with leukopenia (total leukocyte count of less than 4,000/microliter) or thrombocytopenia (platelet count less than 100,000/microliter) and either non-structural protein 1 (NS1) or dengue IgM positive. DF without any evidence of plasma leakage or shock was called as classical DF. DHF was defined as confirm DF with evidence of plasma leakage as indicated by more than $20 \%$ increase or decreases in hematocrit from baseline or radiological evidence of plasma leakage in the form of ascites, pleural effusion, increased gallbladder wall thickness, or pericholecystic fluid. DSS was defined as DF with any sign of shock like hypotension (blood pressure less than $90 / 60 \mathrm{mmHg}$, pulse pressure $<20 \mathrm{mmHg}$, urine output $<0.5 \mathrm{ml} / \mathrm{Kg} / \mathrm{hr}$, or capillary refill time $>2$ seconds).

\section{Ethical approval}

Ethical approval was obtained from the Research and Ethical Committee of Rawalpindi Medical University, Pakistan (Holy Family Hospital is allied with Rawalpindi Medical University). Reference number of approval letter is R-60/RMU.

\section{Sampling}

Sample size was calculated using the MedCalc statistical software version 19.3.1 (MedCalc Software bv, Ostend, Belgium). The minimum sample size calculated was 20 (10 in each group), using reference data from the work of Chhina et al. [9]. After informed consent, 65 patients were included in the study via nonprobability convenience sampling.

\section{Data collection and analysis}

Patients were divided into two groups: group 1 included patients who had classical DF, whereas group 2 included patients with DHF and DSS. All the relevant data were noted, including age, gender, ALT levels at presentation, bleeding manifestations, and development of DHF and DSS over the course of hospital stay. Complete data were entered and then analyzed using IBM SPSS Statistics for Windows, Version 25.0 (Armonk, NY: IBM Corp). Means and standard deviations were calculated for quantitative data, such as age and hospital stay. Frequencies and percentages were computed for qualitative data, such as gender and DF/DHF/DSS. The Mann-Whitney U test was applied to compare mean ALT levels at presentation of both the groups. Null hypothesis formulated for testing stated that mean ALT levels of both the groups were equal. Confidence level was $95 \%$, and p value of less than 0.05 was taken as significant.

\section{Results}

Out of total 65 patients, 45 (69.2\%) were males and 20 (30.8\%) were females. The mean age of the patients was $36.86( \pm 13.9)$ years. Classical DF was present in 18 (27.7\%) patients, whereas $41(63.1 \%)$ patients developed DHF and 6 (9.2\%) patients progressed to DSS. None of the patients included in the study expired. The mean duration of hospital stay was $3.7( \pm 1.2)$ days. Only nine (13.8\%) patients had ALT below $41 \mathrm{U} / \mathrm{L}$ at presentation, and mean level of ALT at presentation in the whole study population was $201.4( \pm 418.46) \mathrm{U} / \mathrm{L}$. Table 1 shows group wise distribution of these parameters. 


\section{Cureus}

\begin{tabular}{|c|c|c|c|}
\hline & Group 1 (DF) & Group 2 (DHF and DSS) & Total (DF, DHF, and DSS) \\
\hline Number of patients (n) & 18 & 47 & 65 \\
\hline Mean age (years) & $32.94( \pm 14.1)$ & $38.36( \pm 13.73)$ & $36.86( \pm 13.9)$ \\
\hline Hospital stay (days) & $3.33( \pm 0.77)$ & $3.89( \pm 1.20)$ & $3.7( \pm 1.20)$ \\
\hline Mean ALT at presentation (U/L) & $131.67( \pm 244.48)$ & 228.15 ( \pm 467.88$)$ & $201.4( \pm 418.46)$ \\
\hline
\end{tabular}

\section{TABLE 1: Group wise distribution of various parameters}

ALT, alanine aminotransferase; DF, dengue fever; DHF, dengue hemorrhagic fever; DSS, dengue shock syndrome

Levels of ALT at presentation in groups 1 and 2 were not normally distributed (kurtosis was 15 and 24.5, respectively, for both groups), and variances of both the groups were also not same as the $\mathrm{p}$ value of Levene's test for homogeneity of variances was $0.524(>0.05)$. Therefore, Mann-Whitney $\mathrm{U}$ test was applied to compare differences between ALT levels of both groups, and $\mathrm{p}$ value was found to be $0.018(<0.05)$ and $\mathrm{Z}$ was -0.2361 . Thus, our null hypothesis was rejected, and it was concluded that there was a statistical difference between ALT levels of both the groups. The effect size was calculated to be 0.087 .

\section{Discussion}

DF is a common viral illness throughout the world [10]. Classical DF carries less mortality and less rate of complications as compared to DHF and DSS, which are more severe forms of illness [11]. Studies have shown that dengue virus affects the reticuloendothelial cells; thus, liver involvement is a well-known feature of dengue virus infection $[12,13]$. The present study found out that only $13.8 \%$ DF patients had normal ALT levels. Studies carried out by Ahmed et al., Kittitrakul et al., and Ashfaq et al. revealed that 38\%, 31.7\%, and 21.4\% of patients had normal ALT levels, respectively [14-16]. The mean ALT level of all the patients was 201.4 U/L in our study. This value was comparable to results obtained by Chhina et al. (218.6 U/L) and Manohar et al. (201.1 U/L) [9,17]. Studies done by Kittitrakul et al. and Srivastava et al. showed mean ALT levels of $152 \mathrm{U} / \mathrm{L}$ and $121 \mathrm{U} / \mathrm{L}$, respectively, which were lower than the levels observed in our study $[16,18]$.

Patients with classical DF in our study had significantly lower mean ALT levels than patients with DHF and DSS. No similar studies were done locally in Pakistan; however, studies done by Chhina et al. (India), Kittitrakul et al. (Thailand), Manohar et al. (India), and Srivastava et al. (India) showed similar results [9,1618].

This study has various limitations, including small sample size, non-probability sampling technique, single center-based experience, and non-inclusion of other markers of hepatic dysfunction. Thus, more large-scale studies are needed to evaluate these findings in detail and see whether other biomarkers of hepatic dysfunction also correlate with dengue severity or not.

\section{Conclusions}

Higher serum levels of ALT at presentation can give a clue about possibility of progression to severe forms of DF (DHF and DSS). Thus, patients with higher ALT at presentation should be prioritized and monitored more rigorously, so that clinical deterioration can be picked at earliest and managed timely.

\section{Additional Information}

\section{Disclosures}

Human subjects: Consent was obtained by all participants in this study. Research and ethical committee, Rawalpindi Medical University, Rawalpindi, Pakistan. issued approval R-60/RMU (12-10-2019). This research project conforms to accepted ethical standards established by Institution Research Forum of Rawalpindi Medical University (RMU). Therefore this research proposal has been approved by Institutional Research Forum and The Research and Ethical Committee of RMU. It is allowed to initiate this research project in RMU. Animal subjects: All authors have confirmed that this study did not involve animal subjects or tissue. Conflicts of interest: In compliance with the ICMJE uniform disclosure form, all authors declare the following: Payment/services info: All authors have declared that no financial support was received from any organization for the submitted work. Financial relationships: All authors have declared that they have no financial relationships at present or within the previous three years with any organizations that might have an interest in the submitted work. Other relationships: All authors have declared that there are no other relationships or activities that could appear to have influenced the submitted work. 


\section{References}

1. Stanaway JD, Shepard DS, Undurraga EA, et al.: The global burden of dengue: an analysis from the Global Burden of Disease study 2013. Lancet Infect Dis. 2016, 16:712-723. 10.1016/S1473-3099(16)00026-8

2. Imai N, Dorigatti I, Cauchemez S, Ferguson NM: Estimating dengue transmission intensity from seroprevalence surveys in multiple countries. PLoS Negl Trop Dis. 2015, 9:e0003719.

10.1371/journal.pntd.0003719

3. Pongpan S, Wisitwong A, Tawichasri C, Patumanond J: Prognostic indicators for dengue infection severity. Int J Clin Pediatr. 2013, 2:12-18. 10.4021/ijcp73w

4. Khan MIH, Anwar E, Agha A, Hassanien NSM, Ullah E, Syed IA, Raja A: Factors predicting severe dengue in patients with dengue fever. Mediterr J Hematol Infect Dis. 2013, 5:e2013014. 10.4084/MJHID.2013.014

5. Ahmed A, Alvi AH, Butt A, Nawaz AA, Hanif A: Assessment of dengue fever severity through liver function tests. J Coll Physicians Surg Pak. 2014, 24:640-644.

6. Villar-Centeno LA, Diaz-Quijano FA, Martinez-Vega RA: Biochemical alterations as markers of dengue haemorrhagic fever. Am J Trop Med Hyg. 2008, 78:370-374. 10.4269/ajtmh.2008.78.370

7. Huerre MR, Lan NT, Marianneau P, et al.: Liver histopathology and biological correlates in five cases of fatal dengue fever in Vietnamese children. Virchows Archive. 2001, 438:107-115. 10.1007/s004280000329

8. Dengue Expert Advisory Group (DEAG), Dengue GCP guidelines 2012. (2012). Accessed: June 19, 2020: http://www.phc.org.pk/downloads/Dengue\%20GCP\%20GUIDELINES.pdf.

9. Chhina RS, Goyal O, Chhina DK, Goyal P, Kumar R, Puri S: Liver function tests in patients with dengue viral infection. Dengue Bull. 2008, 32:110-117.

10. Parkash O, Almas A, Jafri SW, Hamid S, Akhtar J, Alishah H: Severity of acute hepatitis and its outcome in patients with dengue fever in a tertiary care hospital, Karachi, Pakistan (South Asia). BMC Gastroenterol. 2010, 10:43. 10.1186/1471-230X-10-43

11. Chen RF, Yang KD, Wang L, Liu JW, Chiu CC, Chang JT: Different clinical and laboratory manifestations between dengue haemorrhagic fever and dengue fever with bleeding tendency. Trans R Soc Trop Med Hyg. 2007, 101:1106-1113. 10.1016/j.trstmh.2007.06.019

12. Nguyen TL, Nguyen TH, Tieu NT: The impact of dengue haemorrhagic fever on liver function . Res Virol. 1997, 148:273-277. 10.1016/S0923-2516(97)88364-1

13. Mohan B, Patwari AK, Anand VK: Brief report. Hepatic dysfunction in childhood dengue infection . J Trop Pediatr. 2000, 46:40-43. 10.1093/tropej/46.1.40

14. Ahmed SI, Khalid MA, Baqai HZ, Ali SF, Ranja ZA: Dengue fever in Northern Pakistan: the hepatic implications. JRMC. 2009, 13:56-59.

15. Ashfaq MW, Nadeem M, Khalid MA, Shafiq MM, Ahmad SI: Liver biochemistry: difference between dengue fever and non dengue febrile illnesses. JIMDC. 2016, 5:10-13.

16. Kittitrakul C, Silachamroon U, Phumratanaprapin W, Krudsood S, Wilairatana P, Treeprasertsuk S: Liver function tests abnormality and clinical severity of dengue infection in adult patients. J Med Assoc Thai. 2015, 98:S1-S8.

17. Manohar B, Latha NM, Kumar BS, Madhavi K, Sivaramadu K, Dudala SR: Clinical profile and liver function in dengue. NIJP. 2015, 4:15-21.

18. Srivastava G, Chhavi N, Goel A: Validation of serum aminotransferases levels to define severe dengue fever in children. Pediatr Gastroenterol Hepatol Nutr. 2018, 21:289-296. 10.5223/pghn.2018.21.4.289 